PROCEEDINGS OF THE AMERICAN MATHEMATICAL SOCIETY

Volume 124, Number 9, September 1996

\title{
EXTENDING FINITE GROUP ACTIONS FROM SURFACES TO HANDLEBODIES
}

\author{
MARCO RENI AND BRUNO ZIMMERMANN
}

(Communicated by Ronald Stern)

\begin{abstract}
We show that every action of a finite dihedral group on a closed orientable surface $\mathcal{F}$ extends to a 3-dimensional handlebody $\mathcal{V}$, with $\partial \mathcal{V}=$ $\mathcal{F}$. In the case of a finite abelian group $G$, we give necessary and sufficient conditions for a $G$-action on a surface to extend to a compact 3-manifold, or, equivalently in this case, to a 3-dimensional handlebody; in particular all (fixed-point) free actions of finite abelian groups extend to handlebodies. This is no longer true for free actions of arbitrary finite groups: we give a procedure which allows us to construct free actions of finite groups on surfaces which do not extend to a handlebody. We also show that the unique Hurwitz action of order $84(g-1)$ of $P S L(2,27)$ on a surface $\mathcal{F}$ of genus $g=118$ does not extend to any compact 3-manifold $M$ with $\partial M=\mathcal{F}$, thus resolving the only case of Hurwitz actions of type $\operatorname{PSL}(2, q)$ of low order which remained open in an earlier paper (Math. Proc. Cambridge Philos. Soc. 117 (1995), 137-151).
\end{abstract}

\section{INTRODUCTION}

Let $G$ be a finite group of orientation-preserving homeomorphisms of a closed orientable surface $\mathcal{F}$ of genus $g>1$ (a $G$-action on $\mathcal{F}$ ). Then $\mathcal{F} \rightarrow \mathcal{F} / G$ is a branched covering and the quotient orbifold $\mathcal{O}:=\mathcal{F} / G$ is again a closed orientable surface of some genus $\gamma \geq 0$ with finitely many branch points of branching orders $n_{1}, \ldots, n_{\delta}$ (see [8], Proof of the Proposition). We say that $\mathcal{O}$ has signature $\left(\gamma ; n_{1}, \ldots, n_{\delta}\right)$. We can uniformize $\mathcal{O}$ by a Fuchsian group of the same signature (a discrete group of orientation-preserving isometries of the hyperbolic plane $\mathbf{H}^{2}$; see [12], [15] for the theory of Fuchsian groups); this means that $\mathcal{O}$ and $\mathbf{H}^{2} / F$ are homeomorphic as orbifolds. Therefore we may assume that $\mathcal{O}$ is a hyperbolic orbifold $\mathbf{H}^{2} / F$; lifting this hyperbolic structure to $\mathcal{F}$ we may assume that $\mathcal{F}$ is a hyperbolic (or Riemann) surface and that $G$ acts by isometries (or conformal automorphisms). Also, the orbifold fundamental group $\pi_{1} \mathcal{O}$ of $\mathcal{O}$ is isomorphic to $F$, which is the universal covering group of $\mathcal{O}$ (see [10] for basic information about orbifolds). In [8] we gave necessary and sufficient conditions, in terms of its signature, such that the orbifold $\mathcal{O}$ can be uniformized by a finite extension of a Schottky group. In particular, this is the case if the $G$-action on $\mathcal{F}$ extends to a 3 -dimensional handlebody $\mathcal{V}$ of genus $g$ with $\partial \mathcal{V}=\mathcal{F}$, so we get necessary conditions for such an extension. These conditions are not sufficient in general; in fact the possibility of such an extension

Received by the editors February 2, 1995.

1991 Mathematics Subject Classification. Primary 57M60; Secondary 57S25, 30F99.

Key words and phrases. Surface, handlebody, handlebody orbifold, finite group action.

(c)1996 American Mathematical Society 
depends to a great extent on the specific class of finite groups one is considering. In the present paper we consider dihedral and abelian groups. In the case of dihedral groups all actions extend:

Theorem 1. Let $G=D_{n}$ be a dihedral group of order $2 n$ which acts on a closed orientable surface $\mathcal{F}$ of genus $g>1$ by orientation-preserving homeomorphisms. Then the action extends to a handlebody $\mathcal{V}$ with $\partial \mathcal{V}=\mathcal{F}$.

In order to formulate the result for abelian groups, we need some notation. Suppose that the finite group $G$ acts by orientation-preserving isometries on the closed hyperbolic surface $\mathcal{F}$. Then the universal covering of $\mathcal{F}$ is the hyperbolic plane $\mathbf{H}^{2}$ and the covering group is a Fuchsian group $S$ without torsion, $\mathcal{F}=\mathbf{H}^{2} / S$. Let $F$ be the Fuchsian group consisting of all lifts of elements of $G$ to $\mathbf{H}^{2}$. Then the $G$-action on $\mathcal{F}$ is determined by the canonical projection $\pi: F \rightarrow G$, with kernel $S$. Suppose the Fuchsian group $F$ has a signature of the form $\left(\gamma ; n_{1}, \ldots, n_{\alpha}, 2, \ldots, 2\right)$ where $n_{j}>2,1 \leq j \leq \alpha$; then $F$ has a presentation of the form:

$$
\begin{aligned}
\left\langle a_{1}, b_{1}, \ldots, a_{\gamma}, b_{\gamma}, x_{1}, \ldots, x_{\alpha}, y_{1}, \ldots, y_{\beta}\right| \prod_{i=1}^{\gamma}\left[a_{i}, b_{i}\right] \prod_{j=1}^{\alpha} x_{j} \prod_{k=1}^{\beta} y_{k}=1, \\
\left.x_{j}^{n_{j}}=1, j=1, \ldots, \alpha, y_{k}^{2}=1, k=1, \ldots, \beta\right\rangle .
\end{aligned}
$$

Theorem 2. Suppose an action of a finite abelian group $G$ on a closed orientable surface $\mathcal{F}$ of genus $g>1$ is given, determined by a surjection $\pi: F \rightarrow G$ of a Fuchsian group onto $G$. Then the following are equivalent:

(i) The $G$-action on $\mathcal{F}$ extends to a handlebody $\mathcal{V}$, with $\partial \mathcal{V}=\mathcal{F}$.

(ii) The $G$-action on $\mathcal{F}$ extends to a compact 3-manifold $M$, with $\partial M=\mathcal{F}$.

(iii) The generators $x_{1}, \ldots, x_{\alpha}$ of $F$ can be partitioned into pairs $x_{s}, x_{t}$ such that $\pi\left(x_{s}\right)=\pi\left(x_{t}^{-1}\right)$.

Remarks. (a) For finite nonabelian groups $G$, (i) and (ii) of Theorem 2 are not equivalent in general: in [2] it was shown that many Hurwitz actions of type $P S L(2, q)$ extend to compact 3-manifolds; on the other hand, by the necessary condition in [8], no Hurwitz action extends to a handlebody.

(b) The case of finite cyclic groups $G$ or, equivalently, periodic homeomorphisms of surfaces, has been considered in [1, Proposition 3.1]. There it was shown that a periodic diffeomorphism of a surface bounds some compact 3-manifold if and only if the fixed point data cancels. This corresponds to our condition (iii) in Theorem 2. Note that in condition (iii) we don't have to consider elements of order 2 in $F$. The reason is that in a compact 3-orbifold which is a quotient of a 3-manifold by an abelian group, the singular points are of cyclic type $Z_{n}$ or of dihedral type $D_{2}$ (the dihedral group of order 4) because these are the only abelian groups among the possible types (see Figure 1). This implies that a singular axis of order $n$ which starts on the boundary of the 3-orbifold has to end again on the boundary if $n>2$, whereas for $n=2$ it may also end in a dihedral point of type $D_{2}$ (from which then 2 new axes of order 2 emanate).

(c) The main point of the proofs of Theorems 1 and 2 is the construction of the group actions on handlebodies which extend the given actions on surfaces once the 
appropriate condition holds (condition (iii) in Theorem 2, no condition in Theorem 1). This is achieved by constructing appropriate handlebody orbifolds bounding the given quotient 2-orbifolds of the surface actions and then taking regular branched (orbifold-) coverings by handlebodies.

Corollary. Every (fixed point) free action of a finite abelian group on a surface $\mathcal{F}$ extends to a handlebody $\mathcal{V}$.

In general, the elements of the extended action will have fixed points in the interior of $\mathcal{V}$ (in fact, the 2-dimensional bordism group of free bordism classes of free actions of a finite group $G$ on surfaces is isomorphic to the second homology group $H_{2}(G)$, see [13]; see also [14] for an easy algorithm to decide whether a free action of a finite abelian group bounds freely). The Corollary is no longer true for free actions of finite nonabelian groups. In section 3 we give a procedure for how to construct free actions of finite groups on surfaces which do not extend to a handlebody. We do not know if these actions extend to some other compact 3-manifold; in fact, at present we do not know any example of a free action which extends to a compact 3-manifold but not to a handlebody.

In the last section we solve a problem which remained open in [2]. We show that the unique Hurwitz action of order $84(g-1)$ of the group $P S L(2,27)$ on a surface $\mathcal{F}$ of genus $g=118$ does not extend to any compact 3 -manifold $M$ with $\partial M=\mathcal{F}$, thus resolving the only case of Hurwitz actions of type $\operatorname{PSL}(2, q)$ of low order which remained open in [2].

Recently the paper [5] appeared where, in a different but equivalent formulation (uniformization of finite group actions on surfaces by Schottky groups), necessary and sufficient conditions are given for finite cyclic group-actions on surfaces to extend to handlebodies. The methods in [5] are 2-dimensional and thus completely different from the 3 -dimensional methods used in the present work; in fact, it seems that the 3-dimensional approach gives a clearer picture and a better understanding of the situation.

\section{Finite GRoup ACtions ON HANDLEBodies}

In [6] an algebro-geometrical theory of finite group actions on handlebodies has been developed; we recall some terminology from it, adopting a slightly different point of view (see also [8]). Let $G$ be a finite group acting orientation-preservingly on a handlebody $\mathcal{V}$. Let $D$ be a 2-dimensional properly embedded disk in $\mathcal{V}$ such that $\partial D=D \cap \partial \mathcal{V}$ is a nontrivial closed curve on $\mathcal{F}=\partial \mathcal{V}$. By the equivariant loop theorem/Dehn lemma ([7]; note that this is not needed for the proofs of Theorems 1 and 2, however) we can assume that $g(D)=D$ or $g(D) \cap D=\varnothing$, for all $g \in G$. When cutting $\mathcal{V}$ along the system of disjoint disks $G(D)$, that is, removing the interior of a $G$-invariant regular neighbourhood of $G(D)$ (which is a collection of 1-handles, that is, products of a 2-disk with an interval), we get again a collection of handlebodies of lower genus on which $G$ acts. Applying inductively the above procedure of cutting along disks, we finally end up with a collection of disjoint 3-balls on which $G$ acts. Thus the quotient orbifold $\mathcal{H}:=\mathcal{V} / G$ is built up from orbifolds which are quotients of 3-balls by finite groups of homeomorphisms (their stabilizers in $G$ ), connected by finite cyclic quotients of 1-handles, which are the projections of the removed regular neighbourhoods of the disks (first type of orbifold in Figure 1). The finite orientation-preserving groups which can act on the 3-ball or the 2-sphere are the finite subgroups of the orthogonal group $\mathrm{SO}(3)$. It is well 


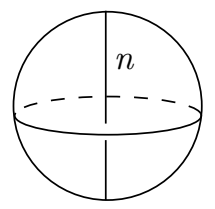

cyclic

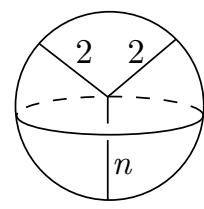

dihedral

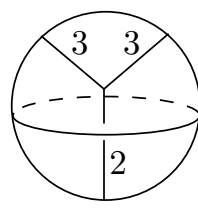

tetrahedral

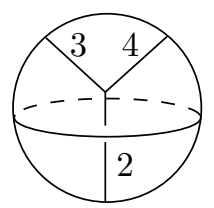

octahedral

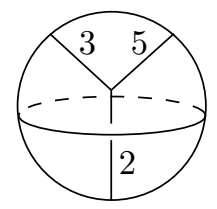

icosahedral

$(0 ; 2,3,5)$

FiguRe 1

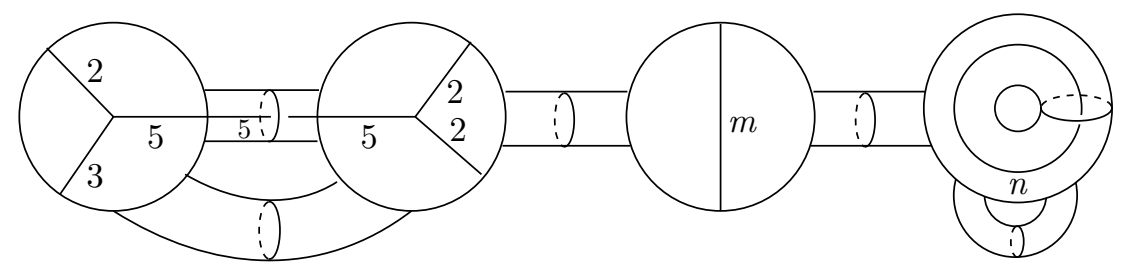

Figure 2

known that, on the boundary of the 3-ball, the actions are standard, i.e. conjugate to orthogonal actions. By Thurston's orbifold geometrization theorem [11] (which again is not needed for the proofs of the theorems), the same is true for the whole 3 -ball. The figures of the possible quotient orbifolds, together with the signatures of the boundaries, are listed in Figure 1; the underlying topological space is the 3-ball in each case.

These quotient orbifolds are connected by the 1-handle orbifolds; the result is called a handlebody orbifold in [6]. An example of a handlebody orbifold is given in Figure 2; in this example the boundary has signature $(3 ; 2,3,2,2, m, m)$. To each handlebody orbifold $\mathcal{H}$ one can associate a graph of groups: the vertices, resp. edges, correspond to the quotients of the 3-balls, resp. 1-handles, and to each vertex, resp. edge, we associate the corresponding finite group, writing only the orders of the nontrivial cyclic groups associated to the edges. Note that, using an orbifold version of Van Kampen's theorem (see [4]), the orbifold fundamental group of $\mathcal{H}$ is isomorphic to the fundamental group of the above graph of groups (see [9] or [15] for definitions about graphs of groups). Deleting the edges whose associated groups are trivial we get exactly the singular set of the handlebody orbifold $\mathcal{H}$. This singular set is a graph $\Gamma$ all of whose vertices have valence 2 or 3 .

More generally, if $G$ is a finite group acting smoothly and orientation-preservingly on a 3-manifold $M$, then using differentials and averaging a scalar product on the tangent space over the finite group $G$, the isotropy group $I_{p} \subset G$ of a point $p \in M$ has a faithful representation in $\mathrm{SO}(3)$; moreover locally the exponential map is an equivariant diffeomorphism, hence the action of $G$ near $p$ is linear and again the singular set of the quotient orbifold $M / G$ is a graph $\Gamma$ as above.

We will start with the

Proof of Theorem 2. The only finite abelian subgroups of $\mathrm{SO}(3)$ are cyclic groups and the dihedral group $D_{2}$ of order 4 . Now (ii) implies (iii) because, in a singular point of order $n>2$ of the 2-orbifold $\mathcal{O}=\mathcal{F} / G$ has to start an axis of order $n$ of 
(i)

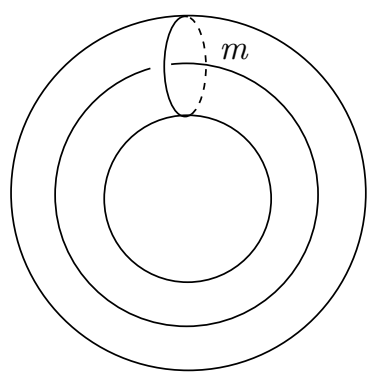

$(m \geq 1)$ (ii)

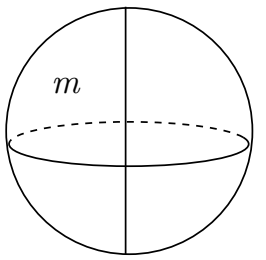

$(m>1)$

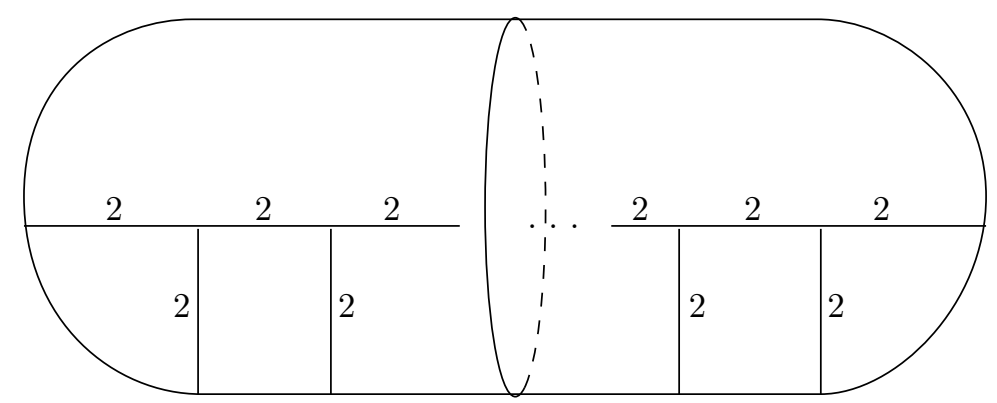

FiguRE 3

the singular set of the 3 -orbifold $M / G$ which has to end in another singular point of order $n>2$ on $\mathcal{O}$.

It follows that the singular points of order $n>2$ on $\mathcal{O}$ are paired; equivalently, the generators $x_{j}$ of $F$ corresponding to these singular points come in pairs as in (iii) of the theorem.

As clearly (i) implies (ii) it remains to show that (iii) implies (i). For this we will construct a handlebody orbifold $\mathcal{H}$ which is a connected sum along 1-handles of pieces of one of the three types in Figure 3, such that $\partial \mathcal{H}=\mathcal{O}=\mathcal{F} / G$ and such that the surjection $\pi: F \cong \pi_{1} \mathcal{O} \rightarrow G$ factors in the following way:

$$
\pi: \pi_{1} \mathcal{O} \rightarrow \pi_{1} \mathcal{H} \stackrel{\psi}{\rightarrow} G
$$

where the first arrow is induced by the inclusion of $\mathcal{O}=\partial \mathcal{H}$ into $\mathcal{H}$; moreover we want the kernel of the surjection $\psi$ to be torsionfree. Then the regular covering of $\mathcal{H}$ corresponding to the kernel of $\psi$ is a handlebody $\mathcal{V}$ on which $G$ acts as the group of covering transformations (see [6], pp. 390,391); on the boundary $\partial \mathcal{V}=\mathcal{F}$ we get our original $G$-action corresponding to the surjection $\pi: \pi_{1} \mathcal{O} \cong F \rightarrow G$.

The handlebody orbifold $\mathcal{H}$ is a connected sum along 1-handles of pieces as in Figure 3 in the following way:

(a) we get $g$ pieces of type (i), one for each pair of generators $a_{i}, b_{i}$ of $F$, where the branching order $m$ in Figure 3(i) is taken as the order $m_{i}$ of $\pi\left(a_{i}\right) \in G$; the orbifold fundamental group of such a piece is

$$
\left\langle\tilde{a}_{i}, \tilde{b}_{i} \mid\left[\tilde{a}_{i}, \tilde{b}_{i}\right]=1, \tilde{a}_{i}^{m_{i}}=1\right\rangle \cong Z \times Z_{m_{i}} ;
$$




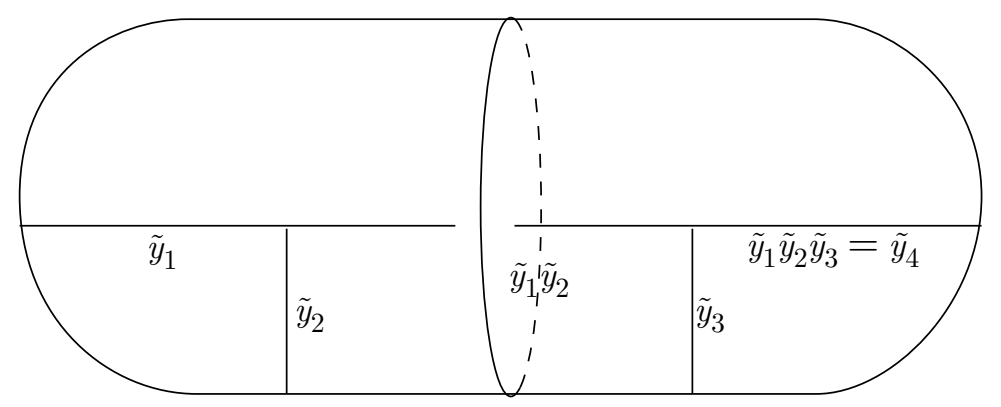

FiguRe 4

(b) we take $\alpha / 2$ pieces of type (ii), one for each pair $x_{s}, x_{t}$ of generators of $F$ such that $\pi\left(x_{s}\right)=\pi\left(x_{t}^{-1}\right)$, where $m$ is the order $m_{s}$ of $\pi\left(x_{s}\right)$ or $\pi\left(x_{t}\right)$ in $G$; the orbifold fundamental group of such a piece is

$$
\left\langle\tilde{x}_{s}, \tilde{x}_{t} \mid \tilde{x}_{t}=\tilde{x}_{s}^{-1}, \tilde{x}_{s}^{m_{s}}=1\right\rangle \cong Z_{m_{s}} ;
$$

using appropriate conjugations of the generators of $F$ we can also assume that corresponding generators $x_{s}$ and $x_{t}$ are adjacent in the defining relation of $F$;

(c) a certain number of pieces of type (iii) (and maybe (ii), with $m=2$, as a special case of (iii)), where the branch points on the boundaries of these pieces correspond to the generators $y_{k}$ of $F$. We start with the first two generators $y_{1}, y_{2}$ of order 2; if the product of $\pi\left(y_{1}\right)$ and $\pi\left(y_{2}\right)$ is trivial we take a piece of type (ii), otherwise the product $\pi\left(y_{1} y_{2}\right)$ has order 2 and we consider the product with $\pi\left(y_{3}\right)$; if this is trivial, we take a piece of type (iii), otherwise $\pi\left(y_{1} y_{2} y_{3}\right)$ has order 2 and we continue with $\pi\left(y_{4}\right)$; each time we get a trivial product we complete a piece of type (iii) and start a new piece with the next generator $y_{k}$ (note that, in general, the singular set of $\mathcal{H}$ is not unique). For example, if it happens for the first time that $\pi\left(y_{1} y_{2} y_{3} y_{4}\right)=1$, then the first piece is as in Figure 4 ; its orbifold fundamental group is

$$
\left\langle\tilde{y}_{1}, \tilde{y}_{2}, \tilde{y}_{3}, \tilde{y}_{4} \mid \tilde{y}_{i}^{2}=1,\left(\tilde{y}_{1} \tilde{y}_{2}\right)^{2}=1,\left(\tilde{y}_{1} \tilde{y}_{2} \tilde{y}_{3}\right)^{2}=1, \tilde{y}_{1} \tilde{y}_{2} \tilde{y}_{3} \tilde{y}_{4}=1\right\rangle \cong D_{2} * z_{2} D_{2},
$$

where the 2 dihedral groups are generated by $\tilde{y}_{1}, \tilde{y}_{2}$, resp. $\tilde{y}_{1} \tilde{y}_{2}, \tilde{y}_{3}$, amalgamated over the cyclic group $Z_{2}$ generated by $\tilde{y}_{1} \tilde{y}_{2}$. Then, by construction, the boundary of the handlebody orbifold $\mathcal{H}$ is a 2 -orbifold which has the same signature $\left(\gamma ; n_{1}, \ldots, n_{\alpha}, 2, \ldots, 2\right)$ as the Fuchsian group $F$. The orbifold fundamental group of $\mathcal{H}$ is isomorphic to the free product of the orbifold fundamental groups of the pieces. The map $\pi_{1} \mathcal{O} \cong F \rightarrow \pi_{1} \mathcal{H}$ induced by the inclusion of $\mathcal{O}=\partial \mathcal{H}$ into $\mathcal{H}$ is given by mapping each generator of $F$ to the same generator with a tilde; then, by our choice of the pieces, it is clear that the surjection $\pi: F \cong \pi_{1} \mathcal{O} \rightarrow G$ factors through $\pi_{1} \mathcal{H}$ as above.

Moreover the kernel of $\psi: \pi_{1} \mathcal{H} \rightarrow G$ is torsionfree because $\psi$ injects the local groups of the singular set of $\mathcal{H}$ into $G$. This finishes the proof of Theorem 2.

Proof of Theorem 1. Let $G=D_{n}$ be the dihedral group of order $2 n$ consisting of $n$ reflections of order 2 and $n$ rotations. The proof is similar to the proof of Theorem 2: we will construct a handlebody orbifold $\mathcal{H}$ such that $\partial \mathcal{H}=\mathcal{O}=\mathcal{F} / G$ and such 


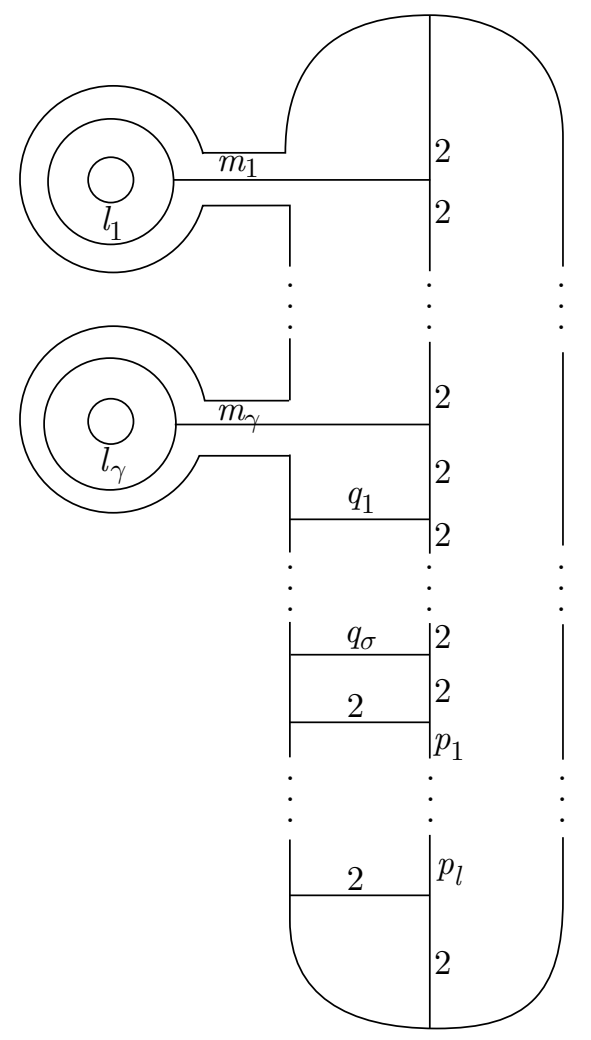

Figure 5

that the surjection $\pi: F \cong \pi_{1} \mathcal{O} \rightarrow G$ factors as

$$
\pi_{1} \mathcal{O} \rightarrow \pi_{1} \mathcal{H} \stackrel{\psi}{\rightarrow} G
$$

Note that among the images $\pi\left(y_{k}\right)$ of the elements of order 2 there is an even number of reflections in $D_{n}$. We distinguish two cases.

(a) Suppose that among the images $\pi\left(y_{k}\right), 1 \leq k \leq \beta$, there are at least two reflections. Then we choose the handlebody orbifold $\mathcal{H}$ as in Figure 5. There are two cases: if $\pi\left(a_{i}\right)$ and $\pi\left(b_{i}\right)$ are both rotations, then $\pi\left(a_{i} b_{i} a_{i}^{-1} b_{i}^{-1}\right)=1$; otherwise one of the generators $a_{i}$ or $b_{i}$, for each $i$, say $a_{i}$, is mapped to a reflection. In Figure 5 , the numbers $l_{i}$, resp. $m_{i}, 1 \leq i \leq \gamma$, denote the orders of $\pi\left(a_{i}\right)$, resp. $\pi\left(a_{i} b_{i} a_{i}^{-1} b_{i}^{-1}\right)$. A number 2 signifies that the corresponding element is mapped by $\pi$ to a reflection in $D_{n}$ (note also that the parts $\left[a_{i}, b_{i}\right], x_{j}$ and $y_{k}$ may be rearranged in any order, by suitable conjugations, in the defining relation of $F \cong \pi_{i} \mathcal{O}$ ). The details are similar to those in the proof of Theorem 2 .

The only other possibility is

(b) There is no reflection among the images $\pi\left(y_{k}\right), 1 \leq k \leq \beta$. Then, because $\pi$ is surjective, one of the other generators $a_{i}$ or $b_{i}$, say $a_{1}$, is mapped to a reflection. Now the handlebody orbifold $\mathcal{H}$ can be chosen as in Figure 6.

This finishes the proof of Theorem 1. 


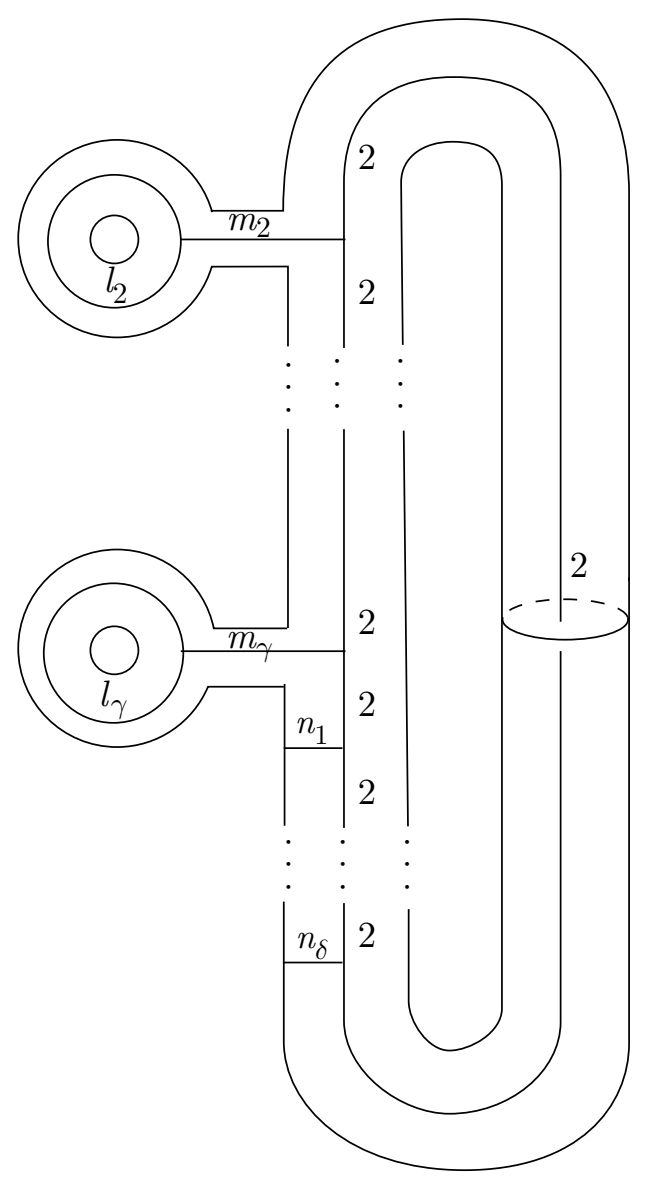

Figure 6

\section{Free aCtions on SURFACES Which do NOT EXTEND}

The Corollary in section 1 is no longer true if we consider arbitrary finite groups.

Proposition 1. Let $F$ be the fundamental group of a surface of genus $\gamma$ and $F^{\prime}$ its commutator subgroup. Let $\pi: F \rightarrow G$ be a surjection onto a finite group $G$ of odd order such that $\pi\left(F^{\prime}\right)$ is an abelian subgroup of $G$ of rank greater than $\gamma(2 \gamma-2)$ (the maximal possibility is $\gamma(2 \gamma-1)-1)$. Then the (free) action of $G$ on a surface $\mathcal{F}$ of genus $g$ determined by the surjection $\pi$ (where $g=|G|(\gamma-1)+1)$ does not extend to a handlebody.

Proof. Suppose the $G$-action on $\mathcal{F}$ extends to a handlebody $\mathcal{V}$, with $\partial \mathcal{V}=\mathcal{F}$. Any subgroup of $G$ has odd order and the only spherical groups of odd order are cyclic groups. Then the quotient $\mathcal{H}=\mathcal{V} / G$ is a handlebody orbifold which is a connected sum along 1-handles of orbifolds of type (i) in Figure 3 (using the equivariant loop theorem/Dehn lemma; see section 2). Its orbifold fundamental group $\pi_{1}(\mathcal{H})$ is a free product $A_{1} * \cdots * A_{\gamma}$ where $A_{i}$ is an abelian group isomorphic to $Z$ or $Z \times Z_{n}$, for $i=1, \ldots, \gamma$, and $Z_{n}$ denotes the integers modulo $n$. In particular we have a 
factorization

$$
\pi: F \rightarrow \pi_{1} \mathcal{H} \stackrel{\psi}{\rightarrow} G
$$

Then $\psi\left(\left(\pi_{1} \mathcal{H}\right)^{\prime}\right)=\pi\left(F^{\prime}\right)$ is an abelian subgroup of $G$. Because $\pi_{1} \mathcal{H} \cong A_{1} * \cdots * A_{\gamma}$, this abelian subgroup is generated by at most $2 \gamma(2 \gamma-1) / 2-\gamma=\gamma(2 \gamma-2)$ elements (all nontrivial commutators of a system of generators of $\pi_{1} \mathcal{H}$ ), in contradiction to our assumption. This finishes the proof of the proposition.

A concrete example of a group $G$ and an epimorphism $\pi$ which verify the hypotheses of Proposition 1 for $\gamma=2$ is constructed in the following way. Fix a prime number $p \geq 3$. Define a group $G^{0}$ as the semidirect product $G^{0}=H K$, $G^{0} / K \cong H$, with $H:=\left(Z_{3}\right)^{2}$ and $K:=\left(Z_{p}\right)^{10}$. Let $h_{1}:=(1,0)$ and $h_{2}:=(0,1)$ be the standard generators of $\left(Z_{3}\right)^{2}$. The generators $h_{1}$, resp. $h_{2}$, act as permutations of the standard generators of $K=\left(Z_{p}\right)^{10}$ given by the permutations of indices $(2,3,4)(5,6,7)(8,9,10)$, resp. $(1,2,3)(4,5,6)(7,8,9)$. We define a homomorphism $\pi: F \rightarrow G^{0}$ as follows:

$$
\begin{array}{ll}
\pi\left(a_{1}\right)=\left(h_{1}, g_{1}\right), & \pi\left(b_{1}\right)=\left(h_{2}, f_{1}\right), \\
\pi\left(a_{2}\right)=\left(h_{1}, g_{2}\right), & \pi\left(b_{2}\right)=\left(h_{2}, f_{2}\right)
\end{array}
$$

where

$$
\begin{gathered}
g_{1}=(1,-1,2,2,0,2,3,1,1,0), \quad g_{2}=(0,-1,1,1,0,1,2,0,1,0), \\
f_{1}=(0,-1,0,2,-3,1,1,-2,0,0), \quad f_{2}=(1,0,1,1,1,0,-1,0,1,1),
\end{gathered}
$$

$g_{1}, g_{2}, f_{1}, f_{2} \in K$; also, $G:=\pi(F) \subset G^{0}$. Then, by a straightforward calculation, the image $\pi\left(F^{\prime}\right)$ of the commutator subgroup of $F$ is an abelian group of rank $\gamma(2 \gamma-1)-1$ (the maximal possibility), so the proposition applies.

\section{Finite group aCtions on SURFaces With FiXed Points}

It is somewhat easier to construct nonextending group actions on surfaces with fixed points because one can use the structure of the singular set in 3-orbifolds. In [2] the case of Hurwitz actions of maximal order 84 $(g-1)$ on closed surfaces of genus $g>1$ is considered. It was noticed that the unique action of the smallest possible Hurwitz group $P S L(2,7)$ of order 168 on the Klein quartic of genus 3 does not extend to any compact 3-manifold $M$. The case of the Hurwitz group $P S L(2,27)$ remained open; it was shown that all other Hurwitz actions of type $\operatorname{PSL}(2, q)$, for $7<q<1000$, do extend. Here we will show that the unique Hurwitz action of $P S L(2,27)$ in fact does not extend. The general conjecture which was verified in [2] for various infinite families is that all Hurwitz actions of type $P S L(2, q)$ extend, with the only exceptions being the groups $P S L(2,7)$ and $P S L(2,27)$; these groups play an exceptional role because the elements of order 7 , resp. 3 , are parabolic, whereas for all other Hurwitz groups of type $\operatorname{PSL}(2, q)$ they are elliptic or hyperbolic.

Proposition 2. The unique Hurwitz action of PSL $(2,27)$ on a surface $\mathcal{F}$ of genus $g=118$ does not extend. In fact there exists a cyclic subgroup of PSL $(2,27)$ which does not extend to any compact 3-manifold $M$.

Proof. We shall indicate two proofs.

(a) Let $(2,3,7)$ denote the triangle group with presentation

$$
\left\langle x, y, t \mid x^{2}=y^{3}=t^{7}=1, x y=t\right\rangle .
$$


As above, the Hurwitz action of $P S L(2,27)$ is determined by a surjection with torsion-free kernel $\pi:(2,3,7) \rightarrow P S L(2,27)$ (see [2]). The element $\pi(y)$ in $P S L(2,27)$ has order 3 and is therefore parabolic (see e.g. [2], Theorem 2.4). By [3], Corollary 2.2 and the remarks following it, $\pi(y)$ is conjugate to an element represented by the matrix

$$
\left(\begin{array}{ll}
1 & 1 \\
0 & 1
\end{array}\right)
$$

or its inverse

$$
\left(\begin{array}{cc}
1 & -1 \\
0 & 1
\end{array}\right)
$$

moreover these two elements are not conjugate because -1 is not a square in the finite field with 27 elements. In particular $\pi(y)$ is not conjugate to its inverse $\pi(y)^{-1}$ in $P S L(2,27)$; therefore $\pi(y)$ does not lie in a dihedral subgroup $D_{3}$ of order 6 , octahedral subgroup $S_{4}$ or dodecahedral subgroup $A_{5}$ of $P S L(2,27)$ (in fact, there are no such subgroups); however it does lie in a tetrahedral subgroup $A_{4}$ of $P S L(2,27)$. Now suppose, by contradiction, that the $P S L(2,27)$-action on $\mathcal{F}$ bounds a compact orientable 3 -manifold $M$, with $\partial M=\mathcal{F}$. Then $M / P S L(2,27)$ is a compact 3 -orbifold with boundary the 2 -orbifold $\mathcal{F} / P S L(2,27)$ which is a 2sphere with three branch points of orders 2,3 and 7 . The singular set of a 3 -orbifold consists of segments, circles and 3-valent graphs where vertices have associated local groups $D_{n}, A_{4}, S_{4}$ or $A_{5}$, while segments and circles have associated cyclic groups (the singular set of the 3 -orbifold $M / P S L(2,27)$ is the projection of the fixed point sets of the nontrivial elements in $P S L(2,27)$, and to each point of the singular set is associated a group which is the stabilizer of a point in the preimage in $M$ under the action; in particular the local groups are subgroups of $\operatorname{PSL}(2,27))$. From the unique branch point of order 3 of $\mathcal{F} / P S L(2,27)$ starts an axis of the singular set of the 3-orbifold, also of order 3. By the above, this axis has to end in a singular point with associated local group $A_{4}$. But then from this point starts a new axis of order 3 (and one of order 2) which has to end in another singular point of type $A_{4}$. Iterating this we get infinitely many points with local group $A_{4}$, contradicting the compactness of the 3 -orbifold $M / P S L(2,27)$.

(b) Now we show that already the cyclic subgroup $Y$ of $P S L(2,27)$ generated by the parabolic element $\pi(y)$ does not bound. By conjugation, we can assume that

$$
\pi(y)=\left(\begin{array}{cc}
1 & \pm 1 \\
0 & 1
\end{array}\right)
$$

Then the normalizer of $Y$ in $P S L(2,27)$ consists of all elements of the form

$$
\left(\begin{array}{cc}
\alpha & \beta \\
0 & \alpha^{-1}
\end{array}\right)
$$

and has $26 \cdot 27 / 2$ elements, therefore the index of $Y$ in its normalizer is 117 . Let $F \subset(2,3,7)$ be the Fuchsian group which is the preimage $\pi^{-1}(Y)$ of $Y$ in the triangle group $(2,3,7)$. The triangle group $(2,3,7)$ has exactly one conjugacy class of cyclic subgroups of order 3; it follows easily that $F$ has 117 conjugacy classes of cyclic subgroups of order 3 and signature $(1 ; 3, \ldots, 3)$ where the number 3 occurs 117 times (the genus is determined by the formula of Riemann-Hurwitz). Then the quotient $\mathcal{F} / Y$ is a torus with 117 branch points of order 3 . Because 117 is an odd number, the 117 branch points cannot be paired by axes of order 3 in a compact 
3-orbifold with boundary $\mathcal{F} / Y$. It follows that the action of $Y$ does not extend. This finishes the proof.

\section{REFERENCES}

1. A. Edmonds and J. Ewing, Remarks on the cobordism group of surface diffeomorphisms, Math. Ann. 259 (1982), 497-504. MR 83m:57026

2. M. Gradolato and B. Zimmermann, Extending finite group actions on surfaces to hyperbolic 3-manifolds, Math. Proc. Cambridge Philos. Soc. 117 (1995), 137-151. MR 96b:57017

3. H. Glover and D. Sjerve, The genus of $P S L_{2}(q)$, J. Reine Angew. Math. 380 (1987), 59-86. MR 89e: 20087

4. A. Haefliger and Quach Ngoc Du, Une présentation du groupe fondamental d'une orbifold, Structure Transverse des Feuilletages, Astérisque 116 (1984), 98-107. MR 86c:57026b

5. R. A. Hidalgo, On Schottky groups with automorphisms, Ann. Acad. Sci. Fenn. 19 (1994), 259-289. MR 95k:30092

6. D. McCullough, A. Miller, and B. Zimmermann, Group actions on handlebodies, Proc. London Math. Soc. (3) 59 (1989), 373-416. MR 90h:57014

7. W. H. Meeks, III and S.-T. Yau, The equivariant loop theorem for three-dimensional manifolds, The Smith Conjecture, Academic Press, New York, 1984, pp. 153-163.

8. M. Reni and B. Zimmermann, Handlebody orbifolds and Schottky uniformizations of hyperbolic 2-orbifolds, Proc. Amer. Math. Soc. 123 (1995), 3907-3914. MR 96b:57016

9. J.-P. Serre, Trees, Springer, 1980. MR 82c:20083

10. W. Thurston, The geometry and topology of three-dimensional manifolds, Lecture Notes, Princeton University, 1978.

11. L Three-manifolds with symmetry, Preprint, 1982.

12. H. Zieschang, E. Vogt, and H.-D. Coldewey, Surfaces and planar discontinuous groups, Lecture Notes in Math., vol. 835, Springer, Berlin, 1980. MR 82h:57002

13. B. Zimmermann, Surfaces and the second homology of a group, Monatsh. Math. 104 (1987), 247-253. MR 89a:57012

14. _ Finite abelian group actions on surfaces, Yokohama Math. J. 38 (1990), 13-21. MR 92c: 57037

15. - Generators and relations for discontinuous groups, Groups and Geometries (A. Barlotti et al., eds.), Kluwer Academic Publishers, 1991, pp. 407-436. MR 93m:20050

Dipartimento di Scienze Matematiche, Università degli Studi di Trieste, 34100 TriESTE, ITALy

E-mail address: reni@univ.trieste.it

E-mail address: zimmer@univ.trieste.it 